\title{
UNIFORM SOBOLEV INEQUALITIES AND ABSOLUTE CONTINUITY OF PERIODIC OPERATORS
}

\author{
ZHONGWEI SHEN AND PEIHAO ZHAO
}

\begin{abstract}
We establish certain uniform $L^{p}-L^{q}$ inequalities for a family of second order elliptic operators of the form $(\mathbf{D}+\mathbf{k}) A(\mathbf{D}+\mathbf{k})^{T}$ on the $d$-torus, where $\mathbf{D}=-i \nabla, \mathbf{k} \in \mathbb{C}^{d}$ and $A$ is a symmetric, positive definite $d \times d$ matrix with real constant entries. Using these Sobolev type inequalities, we obtain the absolute continuity of the spectrum of the periodic Dirac operator on $\mathbb{R}^{d}$ with singular potential. The absolute continuity of the elliptic operator $\operatorname{div}(\omega(\mathbf{x}) \nabla)$ on $\mathbb{R}^{d}$ with a positive periodic scalar function $\omega(\mathbf{x})$ is also studied.
\end{abstract}

\section{INTRODUCTION AND MAIN RESULTS}

Let $\mathbf{D}=\left(D_{1}, D_{2}, \cdots, D_{d}\right)=-i\left(\partial / \partial x_{1}, \partial / \partial x_{2}, \cdots, \partial / \partial x_{d}\right)$ and $A=\left(a_{j l}\right)_{d \times d}$ be a symmetric, positive definite $d \times d$ matrix with real constant entries. We will assume that $d \geq 3$. For $\mathbf{k}=\left(k_{1}, \ldots, k_{d}\right) \in \mathbb{C}^{d}$, we use $(\mathbf{D}+\mathbf{k}) A(\mathbf{D}+\mathbf{k})^{T}$ to denote $\sum_{j, l}\left(D_{j}+k_{j}\right) a_{j l}\left(D_{l}+k_{l}\right)$. Let $\mathbb{T}^{d}=\mathbb{R}^{d} /(2 \pi \mathbb{Z})^{d} \approx[0,2 \pi)^{d}$ be the $d$-torus. Fixing $\mathbf{a}, \mathbf{b} \in \mathbb{R}^{d}$ and $\delta \in \mathbb{R}$, we consider a family of second order elliptic operators

$$
\mathbb{H}_{\rho}=(\mathbf{D}+(\delta+i \rho) \mathbf{a}+\mathbf{b}) A(\mathbf{D}+(\delta+i \rho) \mathbf{a}+\mathbf{b})^{T},
$$

with parameter $\rho \in \mathbb{R}$, on $L^{2}\left(\mathbb{T}^{d}\right)$. Note that $\mathbb{H}_{\rho}^{*}=\mathbb{H}_{-\rho}$.

Let $\|\cdot\|_{p}$ denote the norm in $L^{p}\left(\mathbb{T}^{d}\right)$. In [22], the first named author proved that given $\mathbf{a}, \mathbf{b} \in \mathbb{R}^{d}$ with $|\mathbf{a}|=1$ and $\langle\mathbf{a}, \mathbf{b}\rangle=0$, there exists $\delta \in \mathbb{R}$ such that

$$
\|\psi\|_{q} \leq C\left\|\mathbb{H}_{\rho} \psi\right\|_{p}
$$

for any $\psi \in C^{\infty}\left(\mathbb{T}^{d}\right)$ and any $\rho \in \mathbb{R}$ with $|\rho| \geq 2$, where $C$ is a constant independent of $\psi$ and $\rho$, and $1<p<2<q$ satisfy

$$
\frac{1}{p}+\frac{1}{q}=1, \quad \frac{1}{p}-\frac{1}{q}=\frac{2}{d}
$$

i.e., $p=2 d /(d+2)$ and $q=2 d /(d-2)$. Using inequality (1.2) and L. Thomas's original approach [35], Shen established the absolute continuity of the spectrum of the Schrödinger operator $-\Delta+V(\mathbf{x})$ on $L^{2}\left(\mathbb{R}^{d}\right)$, under the condition that the potential $V$ is real, periodic and $V \in L_{l o c}^{d / 2}\left(\mathbb{R}^{d}\right)[22]$. In the case $d \geq 5$, this improved the earlier results by Reed-Simon [21] and Birman-Suslina [4] on the

Received by the editors July 13, 2005.

2000 Mathematics Subject Classification. Primary 35J10, 42B15.

Key words and phrases. Dirac operator, periodic potential, absolute continuous spectrum, uniform Sobolev inequalities.

The first author was supported in part by the NSF (DMS-0500257). The second author was supported in part by the NSF of Gansu Province, China (ZS021-A25-002-Z) and the NSFC (10371052). 
periodic Schrödinger operator $-\Delta+V$. We remark that in the context of $L^{p}$ spaces, the condition $V \in L_{l o c}^{d / 2}\left(\mathbb{R}^{d}\right)$ is the best possible. Further extensions to the space $L_{l o c}^{d / 2, \infty}$, the Morrey class as well as the Kato class were carried out in [22], [24], [25]. We should point out that the results in [4] are concerned with the more general periodic Schrödinger operators with magnetic potentials. For $d \geq 3$, they rely on the estimates established by A. Sobolev in [30]. We should also mention that there exists an extensive literature on the two-dimensional periodic elliptic operators (see e.g. [1], [2], [20], [4], [5], [10], [19], [23], [24], [26], [27], [28]). For further references on the problem of absolute continuity of periodic operators, we refer the reader to [18] and [34]. More recent work on the subject may be found in [11], [13], [29], [36], [12].

Estimate (1.2) should be considered as a uniform Sobolev inequality on the $d$ torus $\mathbb{T}^{d}$ for the family of second order elliptic operators $\left\{\mathbb{H}_{\rho}: \rho \in \mathbb{R}\right.$ and $\left.|\rho| \geq 2\right\}$. In the setting of $\mathbb{R}^{d}$, similar inequalities had been established earlier by C. Kenig, A. Ruiz and C. Sogge [16] (also see [31] for certain forms of uniform Sobolev inequalities on compact manifolds). Such estimates play a key role in the study of unique continuation properties of differential operators (see e.g. [15]). We remark that the proof of estimate (1.2) in [22] relies on an adaptation of an approach developed in [16] for $\mathbb{R}^{d}$. It also uses the $\left(L^{2}, L^{q}\right)$ bounds of the spectral projection operators for $-\Delta$ on $\mathbb{T}^{d}$ by Sogge [31] in a localization step.

In the first half of this paper we give a different and simpler proof of (1.2). Our new approach is motivated by a work of T. Wolff [37]. Sogge's spectral projection estimates, which play the role of the Fourier restriction theorems, are essential in this approach.

We shall consider an extension of the inequality (1.2). To motivate this extension, we note that operator $\mathbb{H}_{\rho}$ is a multiplier operator. In the region outside of $\{\mathbf{n} \in$ $\left.\mathbb{Z}^{d}: c|\rho| \leq|\mathbf{n}| \leq C|\rho|\right\}$, its multiplier behaves like $(|\mathbf{n}|+|\rho|+1)^{2}$. It is not hard to see that if the multiplier of an operator $T_{\rho}$ behaves like $(|\mathbf{n}|+|\rho|+1)^{2}$ for all $\mathbf{n} \in \mathbb{Z}^{d}$, then

$$
|\rho|^{2}\|\psi\|_{p}+|\rho|\|\nabla \psi\|_{p}+\|\nabla \nabla \psi\|_{p} \leq C\left\|T_{\rho} \psi\right\|_{p}
$$

for any $1<p<\infty$. It follows easily from (1.4) and the Sobolev inequalities as well as complex interpolation that

$$
\|\psi\|_{q} \leq C|\rho|^{d\left(\frac{1}{p}-\frac{1}{q}\right)-2}\left\|T_{\rho} \psi\right\|_{p}
$$

for any exponents $p, q$ satisfying

$$
0 \leq \frac{1}{p}-\frac{1}{q} \leq \frac{2}{d} \text { and } 1<p \leq q<\infty .
$$

Thus in view of (1.5) it seems natural to ask for which pair of exponents $(p, q)$ satisfying (1.6), the inequality

$$
\|\psi\|_{q} \leq C|\rho|^{d\left(\frac{1}{p}-\frac{1}{q}\right)-2}\left\|\mathbb{H}_{\rho} \psi\right\|_{p}
$$

holds. The following is one of the main results of the paper.

Theorem 1.8. There exists a constant $C$ such that for any $\psi \in C^{\infty}\left(\mathbb{T}^{d}\right)$ and any $\rho \in \mathbb{R}$ with $|\rho| \geq 2$, inequality (1.7) holds for all $(p, q)$ in the domain

$$
\left\{(p, q): \frac{1}{q}<\frac{d-1}{2}\left(\frac{1}{p}-\frac{1}{2}\right), \frac{1}{q}<\frac{1}{2}-\frac{2}{d-1}\left(1-\frac{1}{p}\right) \text { and } 0<\frac{1}{p}-\frac{1}{q} \leq \frac{2}{d}\right\} .
$$


Note that if $d=3$, estimate (1.7) holds for any $(p, q)$ satisfying $\frac{1}{2}<\frac{1}{p}-\frac{1}{q} \leq \frac{2}{3}$ and $1<p<q<\infty$. It is also easy to verify that if $\frac{1}{p}-\frac{1}{q}=\frac{2}{d}, 2 d(d-3) /\left(d^{2}-d-4\right)<$ $p<2 d(d-3) /\left(d^{2}-d-8\right)$ and $d \geq 4$, then $(p, q)$ is in the domain (1.9). Thus Theorem 1.8 extends Theorem 6.1 in [22], where it was used to show the absolute continuity of the periodic Schrödinger operators with certain potentials in weak$L^{d / 2}\left(\mathbb{T}^{d}\right)$ space.

It is interesting to point out that estimate (1.4) also implies that

$$
\|\nabla \psi\|_{q} \leq C|\rho|^{d\left(\frac{1}{p}-\frac{1}{q}\right)-1}\left\|T_{\rho} \psi\right\|_{p}
$$

for any $(p, q)$ satisfying $0 \leq \frac{1}{p}-\frac{1}{q} \leq \frac{1}{d}$ and $1<p \leq q<\infty$. However, such an inequality fails for operator $\mathbb{H}_{\rho}$. Instead, we obtain the following.

Theorem 1.11. Suppose $\frac{1}{p}+\frac{1}{q}=1$ and $0 \leq \frac{1}{r}=\frac{1}{p}-\frac{1}{q} \leq \frac{1}{d}$. There exists a constant $C$ such that for any $\psi \in C^{\infty}\left(\mathbb{T}^{d}\right)$ and any $\rho \in \mathbb{R}$ with $|\rho| \geq 2$,

$$
\begin{aligned}
\|\psi\|_{q} & \leq C|\rho|^{-1+\frac{d-1}{2 r}}(\log |\rho|)^{\frac{d+1}{2 r}}\left\|\mathbb{H}_{\rho} \psi\right\|_{p}, \\
\|\nabla \psi\|_{q} & \leq C|\rho|^{\frac{d-1}{2 r}}(\log |\rho|)^{\frac{d+1}{2 r}}\left\|\mathbb{H}_{\rho} \psi\right\|_{p} .
\end{aligned}
$$

The proof of Theorems 1.8 and 1.11 will be given in Section 2. In the second half of this paper, we will use estimate (1.12) to study the spectrum properties of the periodic operators on $\mathbb{R}^{d}$. We obtain the absolute continuity of the periodic Dirac operators with certain singular matrix potentials. We also investigate the problem of absolute continuity of the second order elliptic operator $\operatorname{div}(\omega(\mathbf{x}) \nabla)$, where $\omega$ is a positive periodic scalar function. More precisely, let $V(\mathbf{x})$ be an $m \times m$ real matrix function on $\mathbb{R}^{d}$. Suppose $V$ is selfadjoint and periodic with respect to some lattice of $\mathbb{R}^{d}$. We consider the periodic Dirac operator

$$
\mathcal{D}(V)=\sum_{j=1}^{d} \alpha_{j} D_{j}+V(\mathbf{x}),
$$

acting on functions in $L^{2}\left(\mathbb{R}^{d} ; \mathbb{C}^{m}\right)$. Here $\alpha_{1}, \alpha_{2}, \cdots, \alpha_{d}$ are Hermitian $m \times m$ matrices which satisfy the commutation relations

$$
\alpha_{j} \alpha_{l}+\alpha_{l} \alpha_{j}=2 \delta_{j l} I_{m \times m},
$$

i.e., $\alpha_{j} \alpha_{j}=I_{m \times m}$ and $\alpha_{j} \alpha_{l}=-\alpha_{l} \alpha_{j}$ if $j \neq l$. The following theorem is proved in Section 4.

Theorem 1.15. Let $V=V(\mathbf{x})$ be a real $m \times m$ matrix function on $\mathbb{R}^{d}$. Suppose that $V$ is self-adjoint and periodic with respect to some lattice $\Gamma$ of $\mathbb{R}^{d}$. Also assume that

$$
\alpha_{1} V \alpha_{1}=\alpha_{2} V \alpha_{2}=\cdots=\alpha_{d} V \alpha_{d}
$$

and $V \in \Lambda_{\alpha}^{r, \infty}\left(\mathbb{R}^{d} / \Gamma\right)$ for some $r \geq d$ and $\alpha>(d-1) /(2 r)$, i.e.,

$$
\|V(\cdot+\mathbf{y})-V(\cdot)\|_{L^{r}\left(\mathbb{R}^{d} / \Gamma\right)} \leq C\{\operatorname{dist}(\mathbf{y}, \Gamma)\}^{\alpha},
$$

for any $\mathbf{y} \in \mathbb{R}^{d}$. Then the spectrum of $\mathcal{D}(V)$ on $L^{2}\left(\mathbb{R}^{d} ; \mathbb{C}^{m}\right)$ is absolutely continuous.

Remark 1.18. Since $\Lambda_{\alpha}^{r, \infty} \subset \Lambda_{\alpha^{\prime}}^{r, 2} \subset L_{\alpha^{\prime}}^{r}$ and $L_{\alpha}^{r} \subset \Lambda_{\alpha}^{r, r} \subset \Lambda_{\alpha}^{r, \infty}$ if $r \geq 2$ and $\alpha^{\prime}<\alpha$ [32], it is easy to see that the regularity condition on $V$ in Theorem 1.15 may be restated as $V \in L_{\alpha}^{r}\left(\mathbb{R}^{d} / \Gamma\right)$ for some $r \geq d$ and $\alpha>(d-1) /(2 r)$. 
Remark 1.19. For the Schrödinger operator $-\Delta+V(\mathbf{x})$ on $\mathbb{R}^{3}$ with real periodic potential $V$, L. Thomas [35] proved that the spectrum of the operator is absolutely continuous if $V \in L_{\text {loc }}^{2}\left(\mathbb{R}^{3}\right)$. This was done by using the analytic extension in the so-called quasi-momentum $\mathbf{k}$ and the resolvent estimates for a family of operators of the form $(\mathbf{D}+\mathbf{k})^{2}+V$ on $L^{2}\left(\mathbb{R}^{d} / \Gamma\right)$. There exists an extensive literature based on this approach in the study of absolute continuity of periodic Schrödinger operators and periodic Dirac operators. In particular, for the periodic Dirac operator (1.13) with $V$ satisfying a structure condition similar to (1.16), it was proved by L. Danilov in [6] that the spectrum is absolutely continuous if $V$ is continuous on $\mathbb{R}^{d}$. The result was later extended in [7] to the case $d=3$ and $V \in L_{\text {loc }}^{p}$ for some $p>3$. For $d \geq 4$, the condition on $V$ in [7] was given in terms of Fourier coefficients of $V$. For the periodic Dirac operator with magnetic potential, the absolute continuity was established in [3] (also see [8], [9]). Concerning the regularity condition on the electric potential $V$, we mention that the results in [3] require $V \in L^{r}\left(\mathbb{R}^{2} / \Gamma\right)$ for some $r>2$ if $d=2$, and $V \in C\left(\mathbb{R}^{d} / \Gamma\right)$ if $d \geq 3$. For comparison, we point out that functions in $L_{\alpha}^{r}\left(\mathbb{R}^{d} / \Gamma\right)$ need not be continuous if $\frac{d-1}{2 r}<\alpha \leq \frac{d}{r}$. Indeed, given $0<\beta<\frac{1}{2}+\frac{1}{2 d}$, we may choose $\alpha$ so that $\alpha>\frac{d-1}{2 d}=\frac{1}{2}-\frac{1}{2 d}$ and $\alpha+\beta<1$. It is possible to construct a function in $L_{\alpha}^{d}\left(\mathbb{R}^{d} / \Gamma\right)$ so that

$$
|V(\mathbf{x})| \approx \frac{1}{|\mathbf{x}|^{\beta}} \quad \text { as }|\mathbf{x}| \rightarrow 0 .
$$

Thus our Theorem 1.15 is new for $d \geq 4$.

Let $\omega$ be a positive periodic function in $L^{\infty}\left(\mathbb{R}^{d}\right)$. Suppose $\omega \geq c_{0}$ for some $c_{0}>0$. Consider the second order elliptic operator

$$
\mathcal{L}_{\omega}=\sum_{j, l=1}^{d} D_{j}\left(\omega(\mathbf{x}) a_{j l} D_{l}\right)
$$

on $\mathbb{R}^{d}$, where $A=\left(a_{j l}\right)_{d \times d}$ is a symmetric, positive definite $d \times d$ matrix with real constant entries. It was proved in [23] that if $\omega \in C^{1}\left(\mathbb{R}^{d}\right)$, then the spectrum of $\mathcal{L}_{\omega}$ is absolutely continuous. With estimates (1.12) at our disposal, we are able to establish the absolute continuity of the operator $\mathcal{L}_{\omega}$ for a class of coefficients $\omega(\mathbf{x})$ with discontinuous $\nabla \omega$. More precisely, the following theorem is proved in Section 5 .

Theorem 1.22. Let $\omega(\mathbf{x})$ be a positive, bounded function on $\mathbb{R}^{d}, d \geq 3$. Let $A=$ $\left(a_{j l}\right)_{d \times d}$ be a $d \times d$ symmetric, positive definite matrix with real constant entries. Suppose that $\omega$ is periodic with respect to some lattice of $\mathbb{R}^{d}$ and $\omega(\mathbf{x}) \geq c_{0}$ for some constant $c_{0}>0$. Also assume that $\nabla w \in \Lambda_{\alpha}^{r, \infty}\left(\mathbb{R}^{d} / \Gamma\right)$ for some $r \geq d$ and $\alpha>\frac{d-1}{2 r}$. Then the spectrum of $\mathcal{L}_{\omega}$ is absolutely continuous.

Remark 1.23. For the general second order elliptic operator $\sum_{j, l=1}^{d} D_{j}\left(a_{j l}(\mathbf{x}) D_{l}\right)$ with smooth periodic coefficients, the absolute continuity was established in [13] (also see [36] for the nonsmooth case) under the additional assumption that $a_{j l}$ is symmetric with respect to the hyperplane $x_{1}=0$ in $\mathbb{R}^{d}$. Without this assumption, the problem remains open for $d \geq 3$.

Throughout the rest of this paper, we will assume that $d \geq 3$. We shall use $\|\cdot\|_{p}$ to denote the norm in $L^{p}(\Omega)$ where $\Omega=[0,2 \pi)^{d} \approx \mathbb{T}^{d}=\mathbb{R}^{d} /(2 \pi \mathbb{Z})^{d}$. Finally $C$ and $c$ will be used to denote positive constants which are independent of $\rho$ and $\psi$, and which are not necessarily the same at each occurrence. 


\section{Uniform Sobolev inequalities}

For $\mathbf{k}=\left(k_{1}, k_{2}, \ldots, k_{d}\right) \in \mathbb{C}^{d}$, we consider the second order elliptic operator

$$
\mathbb{H}(\mathbf{k}) \equiv(\mathbf{D}+\mathbf{k}) A(\mathbf{D}+\mathbf{k})^{T}=\sum_{j, l}\left(D_{j}+k_{j}\right) a_{j l}\left(D_{l}+k_{l}\right)
$$

on the $d$-torus $\mathbb{T}^{d}$. For $\psi \in L^{2}\left(\mathbb{T}^{d}\right)$, we may write

$$
\psi(\mathbf{x})=\sum_{\mathbf{n} \in \mathbb{Z}^{d}} \hat{\psi}(\mathbf{n}) e^{i \mathbf{n} \mathbf{x}}
$$

in Fourier series (see e.g. [33]), where

$$
\hat{\psi}(\mathbf{n})=\frac{1}{(2 \pi)^{d}} \int_{\mathbb{T}^{d}} e^{-i \mathbf{n y}} \psi(\mathbf{y}) d \mathbf{y} .
$$

It follows that

$$
\mathbb{H}(\mathbf{k}) \psi(\mathbf{x})=\sum_{\mathbf{n} \in \mathbb{Z}^{d}}(\mathbf{n}+\mathbf{k}) A(\mathbf{n}+\mathbf{k})^{T} \hat{\psi}(\mathbf{n}) e^{i \mathbf{n} \mathbf{x}},
$$

for any $\psi \in C^{2}\left(\mathbb{T}^{d}\right)$. Thus

$$
\{\mathbb{H}(\mathbf{k})\}^{-1} \psi(\mathbf{x})=\sum_{\mathbf{n} \in \mathbb{Z}^{d}} \frac{\hat{\psi}(\mathbf{n}) e^{i \mathbf{n} \mathbf{x}}}{(\mathbf{n}+\mathbf{k}) A(\mathbf{n}+\mathbf{k})^{T}}
$$

is a multiplier operator if $(\mathbf{n}+\mathbf{k}) A(\mathbf{n}+\mathbf{k})^{T} \neq 0$ for any $\mathbf{n} \in \mathbb{Z}^{d}$.

Next we fix $\mathbf{a}=\left(a_{1}, \ldots, a_{d}\right) \in \mathbb{R}^{d}$ and $\mathbf{b}=\left(b_{1}, \ldots, b_{d}\right) \in \mathbb{R}^{d}$. Suppose that $\langle\mathbf{a}, \mathbf{b}\rangle=0$ and $\mathbf{a}$ satisfies

$$
|\mathbf{a}|=1, \quad \mathbf{a} A=\left(s_{0}, 0, \ldots, 0\right) \text { for some } s_{0} \neq 0 .
$$

Since $\mathbf{a} A \mathbf{a}^{T}=s_{0} a_{1}>0$, we know $a_{1} \neq 0$. Let

$$
\delta=\frac{1}{a_{1}}\left(\frac{1}{2}-b_{1}\right)
$$

and

$$
\mathbf{k}=(\delta+i \rho) \mathbf{a}+\mathbf{b},
$$

where $\rho \in \mathbb{R}$ is a parameter. A direct computation shows

$$
\begin{aligned}
& (\mathbf{n}+\mathbf{k}) A(\mathbf{n}+\mathbf{k})^{T}=(\mathbf{n}+\mathbf{b}) A(\mathbf{n}+\mathbf{b})^{T}+2 \delta\left(n_{1}+b_{1}\right) s_{0} \\
& \quad+\left(\delta^{2}-\rho^{2}\right) a_{1} s_{0}+2 i \rho\left(n_{1}+\frac{1}{2}\right) s_{0} .
\end{aligned}
$$

From this, it is easy to verify that if $|\rho| \geq 2$ and $\mathbf{n} \in \mathbb{Z}^{d}$, then

$$
\left|(\mathbf{n}+\mathbf{k}) A(\mathbf{n}+\mathbf{k})^{T}\right| \approx\left|(\mathbf{n}+\mathbf{b}) A(\mathbf{n}+\mathbf{b})^{T}-\rho^{2} a_{1} s_{0}\right|+\left|\rho\left(n_{1}+\frac{1}{2}\right)\right| .
$$

From now on we will assume that $|\rho| \geq 2$. We emphasize again that all constants in this paper are independent of the parameter $\rho$.

Let $B=\sqrt{A}$. Then $(\mathbf{n}+\mathbf{b}) A(\mathbf{n}+\mathbf{b})^{T}=|(\mathbf{n}+\mathbf{b}) B|^{2}$. Using (2.10), it is not hard to see that there exist constants $C>0, c>0$ such that

$$
\begin{aligned}
& \left|(\mathbf{n}+\mathbf{k}) A(\mathbf{n}+\mathbf{k})^{T}\right| \approx \\
& \quad\left\{\begin{array}{cl}
(|\mathbf{n}|+|\rho|)^{2} & \text { if }|\mathbf{n}| \geq C|\rho| \text { or }|\mathbf{n}| \leq c|\rho|, \\
|\rho|\left\{|| \mathbf{n} B|-| \rho\left|\sqrt{a_{1} s_{0}}\right|+\left|n_{1}\right|+1\right\} & \text { if } c|\rho|<|\mathbf{n}|<C \rho .
\end{array}\right.
\end{aligned}
$$


Note that the multiplier $(\mathbf{n}+\mathbf{k}) A(\mathbf{n}+\mathbf{k})^{T}$ behaves well in the region $\left\{\mathbf{n} \in \mathbb{Z}^{d}\right.$ : $|\mathbf{n}| \geq C|\rho|$ or $|\mathbf{n}|<c|\rho|\}$. The following two lemmas will be used to deal with the remaining region $\left\{\mathbf{n} \in \mathbb{Z}^{d}: c|\rho|<|\mathbf{n}|<C|\rho|\right\}$.

Lemma 2.12. For any $p>1$, we have

$$
\sum_{c|\rho|<|\mathbf{n}|<C|\rho|} \frac{1}{\left|(\mathbf{n}+\mathbf{k}) A(\mathbf{n}+\mathbf{k})^{T}\right|^{p}} \leq C_{p}\left\{\begin{array}{cl}
|\rho|^{d-4} \log |\rho| & \text { if } p=2, \\
|\rho|^{d-2 p} & \text { if } p<2, \\
|\rho|^{d-2-p} & \text { if } p>2 .
\end{array}\right.
$$

Proof. It follows from the proof of Lemma 3.2 in [22] (see the estimate of $I_{2}$ on pp. 16-17) that

$$
\sum_{c|\rho|<|\mathbf{n}|<C|\rho|} \frac{1}{\left|(\mathbf{n}+\mathbf{k}) A(\mathbf{n}+\mathbf{k})^{T}\right|^{p}} \leq C|\rho|^{d-2 p} \int_{0}^{C} \frac{d r}{\left\{r+\frac{1}{|\rho|}\right\}^{p-1}} .
$$

From this, one may deduce estimate (2.13) easily.

Lemma 2.15. For any $p>1$ and $\ell \geq 1$, we have

$$
\sum_{\substack{|\mathbf{n} B| \in[\ell, \ell+1) \\ c|\rho|<|\mathbf{n}|<C|\rho|}} \frac{1}{\left|(\mathbf{n}+\mathbf{k}) A(\mathbf{n}+\mathbf{k})^{T}\right|^{p}} \leq \frac{C_{p}|\rho|^{d-p-2}}{\left\{|\ell-| \rho\left|\sqrt{a_{1} s_{0}}\right|+1\right\}^{p-1}} .
$$

Proof. We may assume that $\ell \approx|\rho|$. Using (2.11), we have

$$
\begin{aligned}
& \sum_{\substack{|\mathbf{n} B| \in[\ell, \ell+1) \\
c|\rho|<|\mathbf{n}|<C|\rho|}} \frac{1}{\left|(\mathbf{n}+\mathbf{k}) A(\mathbf{n}+\mathbf{k})^{T}\right|^{p}} \\
& \leq C|\rho|^{-p} \sum_{|\mathbf{n} B| \in[\ell, \ell+1)} \frac{1}{\left\{|| \mathbf{n} B|-| \rho\left|\sqrt{a_{1} s_{0}}\right|+\left|n_{1}\right|+1\right\}^{p}} \\
& \leq C|\rho|^{-p} \int_{\ell-2 \leq|\mathbf{x}| \leq \ell+2} \frac{d \mathbf{x}}{\left\{|| \mathbf{x}|-| \rho\left|\sqrt{a_{1} s_{0}}\right|+\left|x_{1}\right|+1\right\}^{p}} \\
& \leq C|\rho|^{d-2 p} \int_{\frac{\ell-2}{|\rho|} \leq|\mathbf{y}| \leq \frac{\ell+2}{|\rho|}} \frac{d \mathbf{y}}{\left\{|| \mathbf{y}\left|-\sqrt{a_{1} s_{0}}\right|+\left|y_{1}\right|+\frac{1}{|\rho|}\right\}^{p}} \\
& \leq C|\rho|^{d-2 p} \int_{\frac{\ell-2}{|\rho|} \leq r \leq \frac{\ell+2}{|\rho|}} d r \int_{0}^{\pi / 2} \frac{(\sin \theta)^{d-2} d \theta}{\left\{\left|r-\sqrt{a_{1} s_{0}}\right|+r \cos \theta+\frac{1}{|\rho|}\right\}^{p}} \\
& \leq C|\rho|^{d-2 p} \int_{\frac{\ell-2}{|\rho|} \leq r \leq \frac{\ell+2}{\rho \rho \mid}} \frac{d r}{\left\{\left|r-\sqrt{a_{1} s_{0}}\right|+\frac{1}{|\rho|}\right\}^{p-1}} \\
& \leq \frac{C|\rho|^{d-2 p-1}}{\left\{\left|\frac{\ell}{|\rho|}-\sqrt{a_{1} s_{0}}\right|+\frac{1}{|\rho|}\right\}^{p-1}} \\
& =\frac{C|\rho|^{d-p-2}}{\left\{|\ell-| \rho\left|\sqrt{a_{1} s_{0}}\right|+1\right\}^{p-1}} \text {. }
\end{aligned}
$$

The proof is finished. 
We now choose $\eta \in C^{\infty}((0, \infty))$ such that $0 \leq \eta(t) \leq 1$ for any $t>0, \eta(t)=1$ for $c \leq t \leq C$ and $\operatorname{supp}(\eta) \subset(c / 2,2 C)$. Write $\{\mathbb{H}(\mathbf{k})\}^{-1}=T_{1}+T_{2}$, where

$$
\begin{aligned}
& T_{1} \psi=\sum_{\mathbf{n} \in \mathbb{Z}^{d}} \frac{\eta\left(\frac{|\mathbf{n}|}{\rho}\right) \hat{\psi}(\mathbf{n}) e^{i \mathbf{n x}}}{(\mathbf{n}+\mathbf{k}) A(\mathbf{n}+\mathbf{k})^{T}}, \\
& T_{2} \psi=\sum_{\mathbf{n} \in \mathbb{Z}^{d}} \frac{\left(1-\eta\left(\frac{|\mathbf{n}|}{\rho}\right)\right) \hat{\psi}(\mathbf{n}) e^{i \mathbf{n x}}}{(\mathbf{n}+\mathbf{k}) A(\mathbf{n}+\mathbf{k})^{T}} .
\end{aligned}
$$

In view of (2.11), the estimates of operator $T_{2}$ are standard. Indeed, by Hörmander's Multiplier Theorem and the transference theorem (see e.g. [14, Theorem 3.6.7, p. 221]), one has $\left\|T_{2} \psi\right\|_{p} \leq C|\rho|^{-2}\|\psi\|_{p},\left\|\nabla T_{2} \psi\right\|_{p} \leq C|\rho|^{-1}\|\psi\|_{p}$, and $\left\|\nabla \nabla T_{2} \psi\right\|_{p}$ $\leq C\|\psi\|_{p}$ for any $1<p<\infty$. This, together with Sobolev's imbedding and the Riesz-Thorin interpolation theorem, gives the following.

Lemma 2.18. Let $1<p \leq q<\infty$. Then

$$
\begin{gathered}
\left\|T_{2} \psi\right\|_{q} \leq C|\rho|^{d\left(\frac{1}{p}-\frac{1}{q}\right)-2}\|\psi\|_{p} \quad \text { if } \quad \frac{1}{p}-\frac{1}{q} \leq \frac{2}{d}, \\
\left\|\nabla T_{2} \psi\right\|_{q} \leq C|\rho|^{d\left(\frac{1}{p}-\frac{1}{q}\right)-1}\|\psi\|_{p} \quad \text { if } \quad \frac{1}{p}-\frac{1}{q} \leq \frac{1}{d} .
\end{gathered}
$$

The estimates of operator $T_{1}$ are much more involved. The approach we use here is motivated by [37]. It relies on Sogge's spectral projection estimates for elliptic operators on compact manifolds.

Let

$$
q_{0}=\frac{2(d+1)}{d-1}, \quad p_{0}=q_{0}^{\prime}=\frac{2(d+1)}{d+3} .
$$

Lemma 2.20. Suppose $q_{0}<q<\infty$. Then

$$
\begin{aligned}
\left\|T_{1} \psi\right\|_{q} & \leq C|\rho|^{d\left(\frac{1}{p_{0}}-\frac{1}{q}\right)-2}\|\psi\|_{p_{0}}, \\
\left\|\nabla T_{1} \psi\right\|_{q} & \leq C|\rho|^{d\left(\frac{1}{p_{0}}-\frac{1}{q}\right)-1}\|\psi\|_{p_{0}} .
\end{aligned}
$$

Proof. First we write

$$
T_{1} \psi=\sum_{\ell=0}^{\infty} \sum_{\substack{\mathbf{n} \in \mathbb{Z}^{d} \\|\mathbf{n} B| \in[\ell, \ell+1)}} \frac{\eta\left(\frac{|\mathbf{n}|}{\rho}\right) \hat{\psi}(\mathbf{n}) e^{i \mathbf{n x}}}{(\mathbf{n}+\mathbf{k}) A(\mathbf{n}+\mathbf{k})^{T}} .
$$

Next we consider the second order elliptic operator $P=\mathbf{D} A \mathbf{D}^{T}$ on the $d$-torus $\mathbb{T}^{d}$. Note that $P$ has a complete set of eigenfunctions $\left\{e^{i \mathbf{n x}}, \mathbf{n} \in \mathbb{Z}^{d}\right\}$ with the corresponding eigenvalues $\left\{\mathbf{n} A \mathbf{n}^{T}, \mathbf{n} \in \mathbb{Z}^{d}\right\}$. Thus

$$
\zeta_{\ell} \psi=\sum_{\substack{\mathbf{n} \in \mathbb{Z}^{d} \\ \mathbf{n} A \mathbf{n}^{T} \in\left[\ell^{2},(\ell+1)^{2}\right)}} \hat{\psi}(\mathbf{n}) e^{i \mathbf{n} \mathbf{x}}=\sum_{\substack{\mathbf{n} \in \mathbb{Z}^{d} \\|\mathbf{n} B| \in[\ell, \ell+1)}} \hat{\psi}(\mathbf{n}) e^{i \mathbf{n} \mathbf{x}}
$$

is the projection of $\psi$ to the subspace of $L^{2}\left(\mathbb{T}^{d}\right)$ spanned by the eigenfunctions of $P$ with eigenvalues in $\left[\ell^{2},(\ell+1)^{2}\right)$. It then follows from a general result of C. Sogge in $\left[31\right.$, Theorem 2.2(i), p. 127] that for any $\psi \in L^{2}\left(\mathbb{T}^{d}\right)$,

$$
\left\|\zeta_{\ell} \psi\right\|_{q} \leq C(\ell+1)^{\delta(q)}\|\psi\|_{2}
$$


where $2 \leq q \leq \infty$ and

$$
\delta(q)=\left\{\begin{aligned}
d\left|\frac{1}{2}-\frac{1}{q}\right|-\frac{1}{2}, & \text { if } q_{0} \leq q \leq \infty, \\
\frac{(d-1)}{2}\left|\frac{1}{2}-\frac{1}{q}\right|, & \text { if } 2 \leq q \leq q_{0} .
\end{aligned}\right.
$$

By duality, one also has

$$
\left\|\zeta_{\ell} \psi\right\|_{2} \leq C(\ell+1)^{\delta\left(p^{\prime}\right)}\|\psi\|_{p}, \quad 1 \leq p \leq 2 .
$$

Note that for $q_{0}=\frac{2(d+1)}{d-1}$, we have $\delta\left(q_{0}\right)=\frac{1}{q_{0}}$ by $(2.25)$. It follows from $(2.24)$ that

$$
\left\|\sum_{\substack{\mathbf{n} \in \mathbb{Z}^{d} \\|\mathbf{n} B| \in[\ell, \ell+1)}} c_{\mathbf{n}} e^{i \mathbf{n} \mathbf{x}}\right\|_{q_{0}} \leq C(\ell+1)^{\frac{1}{q_{0}}}\left(\sum_{\substack{\mathbf{n} \in \mathbb{Z}^{d} \\|\mathbf{n} B| \in[\ell, \ell+1)}}\left|c_{\mathbf{n}}\right|^{2}\right)^{1 / 2} .
$$

By complex interpolation, estimate (2.27), together with the trivial estimate

$$
\left\|\sum_{\substack{\mathbf{n} \in \mathbb{Z}^{d} \\|\mathbf{n} B| \in[\ell, \ell+1)}} c_{\mathbf{n}} e^{i \mathbf{n x}}\right\|_{\infty} \leq C \sum_{\substack{\mathbf{n} \in \mathbb{Z}^{d} \\|\mathbf{n} B| \in[\ell, \ell+1)}}\left|c_{\mathbf{n}}\right|,
$$

gives

$$
\left\|\sum_{\substack{\mathbf{n} \in \mathbb{Z}^{d} \\|\mathbf{n} B| \in[\ell, \ell+1)}} c_{\mathbf{n}} e^{i \mathbf{n x}}\right\|_{q} \leq C(\ell+1)^{\frac{1}{q}}\left(\sum_{\substack{\mathbf{n} \in \mathbb{Z}^{d} \\|\mathbf{n} B| \in[\ell, \ell+1)}}\left|c_{\mathbf{n}}\right|^{p}\right)^{\frac{1}{p}}
$$

where

$$
q_{0} \leq q \leq \infty, \quad \frac{1}{p}=1-\frac{q_{0}}{2 q} .
$$

Thus, by Minkowski's inequality, estimate (2.29) and Hölder's inequality, for any $q \in\left(q_{0}, \infty\right)$ and $p<2$ given by $(2.30)$, we have

$$
\begin{aligned}
& \left\|T_{1} \psi\right\|_{q} \leq \sum_{c|\rho|<\ell<C|\rho|}\left\|\sum_{\substack{\left.\mathbf{n} \in \mathbb{Z}^{d} \\
|\mathbf{n} B| \in \ell \ell, \ell+1\right)}} \frac{\eta\left(\frac{|\mathbf{n}|}{|\rho|}\right) \hat{\psi}(\mathbf{n}) e^{i \mathbf{n x}}}{(\mathbf{n}+\mathbf{k}) A(\mathbf{n}+\mathbf{k})^{T}}\right\|_{q} \\
& \leq C|\rho|^{\frac{1}{q}} \sum_{c|\rho|<\ell<C|\rho|}\left\{\sum_{\substack{\mathbf{n} \in \mathbb{Z}^{d} \\
|\mathbf{n} B| \in[\ell, \ell+1)}}\left|\frac{|\hat{\psi}(\mathbf{n})|}{(\mathbf{n}+\mathbf{k}) A(\mathbf{n}+\mathbf{k})^{T}}\right|^{p}\right\}^{1 / p} \\
& \leq C|\rho|^{\frac{1}{q}} \sum_{c|\rho|<\ell<C|\rho|}\left\{\sum_{\substack{\mathbf{n} \in \mathbb{Z}^{d} \\
|\mathbf{n} B| \in[\ell, \ell+1)}}|\hat{\psi}(\mathbf{n})|^{2}\right\}^{1 / 2} \\
& \times\left\{\sum_{\substack{\mathbf{n} \in \mathbb{Z}^{d} \\
|\mathbf{n} B| \in[\ell, \ell+1)}} \sum_{\left|(\mathbf{n}+\mathbf{k}) A(\mathbf{n}+\mathbf{k})^{T}\right|^{\frac{2 p}{2-p}}}\right\}^{\frac{2-p}{2 p}} \\
& \leq C|\rho|^{\frac{1}{q}+\frac{1}{q_{0}}+(d-2)\left(\frac{1}{p}-\frac{1}{2}\right)-1}\|\psi\|_{p_{0}} \sum_{c|\rho|<\ell<C|\rho|} \frac{1}{\left\{|\ell-| \rho \mid \sqrt{a_{1} s_{0} \mid}+1\right\}^{\frac{3}{2}-\frac{1}{p}}}
\end{aligned}
$$


where we also used estimates (2.26) and (2.16) in the last inequality. Note that if $0 \leq \alpha<1$,

$$
\begin{aligned}
\sum_{c|\rho| \leq \ell \leq C|\rho|} \frac{1}{\left\{|\ell-| \rho\left|\sqrt{a_{1} s_{0}}\right|+1\right\}^{\alpha}} & \leq C \int_{c|\rho|}^{C|\rho|} \frac{d t}{\left\{|t-| \rho\left|\sqrt{a_{1} s_{0}}\right|+1\right\}^{\alpha}} \\
& \leq C|\rho|^{1-\alpha} \int_{0}^{C} \frac{d t}{\left\{t+\frac{1}{|\rho|}\right\}^{\alpha}} \\
& \leq C|\rho|^{1-\alpha} .
\end{aligned}
$$

We obtain

$$
\begin{aligned}
\left\|T_{1} \psi\right\|_{q} & \leq C|\rho|^{\frac{1}{p}+\frac{1}{q_{0}}+(d-1)\left(\frac{1}{p}-\frac{1}{2}\right)-1}\|\psi\|_{p_{0}} \\
& =C|\rho|^{d\left(\frac{1}{p_{0}}-\frac{1}{q}\right)-2}\|\psi\|_{p_{0}} .
\end{aligned}
$$

The proof of the estimate for $\nabla T_{1}$ in $(2.21)$ is similar.

Remark 2.32. If we let $q=q_{0}$ and $p=2$ in the proof above, the same argument would give

$$
\begin{aligned}
\left\|T_{1} \psi\right\|_{q_{0}} & \leq C|\rho|^{\frac{2}{q_{0}}-1} \log |\rho|\|\psi\|_{p_{0}}, \\
\left\|\nabla T_{1} \psi\right\|_{q_{0}} & \leq C|\rho|^{\frac{2}{q_{0}}} \log |\rho|\|\psi\|_{p_{0}} .
\end{aligned}
$$

The logarithmic function arises in (2.31) when $\alpha=1$.

We need another lemma before we carry out the proof of Theorem 1.8.

Lemma 2.34. For any $1<p<2$, we have

$$
\begin{aligned}
\left\|T_{1} \psi\right\|_{\infty} & \leq C|\rho|^{\frac{d}{p}-2}\|\psi\|_{p}, \\
\left\|\nabla T_{1} \psi\right\|_{\infty} & \leq C|\rho|^{\frac{d}{p}-1}\|\psi\|_{p} .
\end{aligned}
$$

Proof. Let $1<p<2$. By Hölder's inequality,

$$
\begin{aligned}
\left\|T_{1} \psi\right\|_{\infty} & \leq \sum_{\substack{\mathbf{n} \in \mathbb{Z}^{d} \\
c|\rho|<|\mathbf{n}|<C|\rho|}}\left|\frac{\hat{\psi}(\mathbf{n})}{\mid(\mathbf{n}+\mathbf{k}) A(\mathbf{n}+\mathbf{k})^{T}}\right| \\
& \leq\left(\sum_{\mathbf{n} \in \mathbb{Z}^{d}}|\hat{\psi}(\mathbf{n})|^{p^{\prime}}\right)^{\frac{1}{p^{\prime}}}\left(\sum_{\substack{\mathbf{n} \in \mathbb{Z}^{d} \\
c|\rho|<|\mathbf{n}|<C|\rho|}} \frac{1}{\left|(\mathbf{n}+\mathbf{k}) A(\mathbf{n}+\mathbf{k})^{T}\right|^{p}}\right)^{\frac{1}{p}} \\
& \leq C|\rho|^{\frac{d-2 p}{p}}\|\psi\|_{p},
\end{aligned}
$$

where we have used the Hausdorff-Young inequality and estimate (2.13) in the last step. The proof of the second inequality in (2.35) is similar.

We are now ready to give the proof of Theorems 1.8 and 1.11 .

Proof of Theorem 1.8. For $j=1,2$, consider the set $\mathcal{A}_{j}$ of $\left(\frac{1}{p}, \frac{1}{q}\right) \in[0,1] \times[0,1]$ for which the estimate

$$
\left\|T_{j} \psi\right\|_{q} \leq C|\rho|^{d\left(\frac{1}{p}-\frac{1}{q}\right)-2}\|\psi\|_{p}
$$

holds uniformly for $\rho \in \mathbb{R}$ with $|\rho| \geq 2$. Recall that $T_{j}^{*}$ may be obtained simply by replacing the parameter $\rho$ in $T_{j}$ with $-\rho$. By duality, this implies that if $(t, s) \in \mathcal{A}_{j}$, 
then $(s, t) \in \mathcal{A}_{j}$. Also, by the Riesz-Thorin Interpolation Theorem, $\mathcal{A}_{j}$ is a convex set.

Next we note that by Lemmas 2.20 and $2.34, \mathcal{A}_{1}$ contains the set $\{(t, s): t=$ $\frac{1}{p_{0}}$ and $\left.0<s<\frac{1}{q_{0}}\right\}$ and the set $\left\{(t, s): \frac{1}{2}<t<1\right.$ and $\left.s=0\right\}$. It follows that $\mathcal{A}_{1}$ contains the open polygon with vertices at $\left(\frac{1}{2}, 0\right),(1,1),\left(1, \frac{1}{2}\right)$, and $\left(\frac{1}{p_{0}}, \frac{1}{q_{0}}\right)$. In view of Lemma $2.18, \mathcal{A}_{2}$ contains the set $\left\{(t, s) \in(0,1) \times(0,1): 0 \leq t-s \leq \frac{2}{d}\right\}$.

Finally it follows from $\{\mathbb{H}(\mathbf{k})\}^{-1}=T_{1}+T_{2}$ that

$$
\left\|\{\mathbb{H}(\mathbf{k})\}^{-1} \psi\right\|_{q} \leq C|\rho|^{d\left(\frac{1}{p}-\frac{1}{q}\right)-2}\|\psi\|_{p}
$$

holds for any $\left(\frac{1}{p}, \frac{1}{q}\right) \in \mathcal{A}_{1} \cap \mathcal{A}_{2}$. Since $\mathcal{A}_{1} \cap \mathcal{A}_{2}$ contains the set (1.9), we are done.

Proof of Theorem 1.11. In view of the stronger estimates for $T_{2}$ in Lemma 2.18, it suffices to show that

$$
\begin{aligned}
\left\|T_{1} \psi\right\|_{q} & \leq C|\rho|^{-1+\frac{d-1}{2 r}}(\log |\rho|)^{\frac{d+1}{2 r}}\|\psi\|_{p}, \\
\left\|\nabla T_{1} \psi\right\|_{q} & \leq C|\rho|^{\frac{d-1}{2 r}}(\log |\rho|)^{\frac{d+1}{2 r}}\|\psi\|_{p},
\end{aligned}
$$

where $\frac{1}{p}+\frac{1}{q}=1$ and $0 \leq \frac{1}{r}=\frac{1}{p}-\frac{1}{q} \leq \frac{1}{d}$. Clearly, estimate (2.38) holds for $p=q=2$ and $r=\infty$. By Remark 2.32, it holds for $p=p_{0}, q=q_{0}$ and $r=$ $(d+1) / 2$. Consequently, by complex interpolation, estimate $(2.38)$ holds for $p=q^{\prime}$ and $(d+1) / 2 \leq r \leq \infty$. The desired estimate then follows since $d \geq(d+1) / 2$ for $d \geq 2$,

\section{An Approximation PRoperty fOR the Lipschitz SPACE $\Lambda_{\alpha}^{r, \infty}\left(\mathbb{T}^{d}\right)$}

In the rest of this paper, we will use the uniform Sobolev inequalities established in Section 2 to investigate the problem of absolute continuity of periodic operators. Since our regularity assumption on the coefficients of such operators will be given in terms of the modulus of continuity, we need an approximation property for the Lipschitz space $\Lambda_{\alpha}^{r, \infty}\left(\mathbb{T}^{d}\right)$. Recall that for $0<\alpha<1$ and $1 \leq r \leq \infty$, we say $f \in \Lambda_{\alpha}^{r, \infty}\left(\mathbb{T}^{d}\right)$ if $f \in L^{r}\left(\mathbb{T}^{d}\right)$ and there exists a constant $C>0$ such that

$$
\|f(\cdot+\mathbf{y})-f(\cdot)\|_{r} \leq C\left\{\operatorname{dist}\left(\mathbf{y},(2 \pi \mathbb{Z})^{d}\right)\right\}^{\alpha},
$$

for any $\mathbf{y} \in \mathbb{T}^{d}$. For systematic treatment of the space $\Lambda_{\alpha}^{p, q}\left(\mathbb{R}^{d}\right)$, we refer the reader to [32, Chapter 5].

Proposition 3.2. Suppose $f \in \Lambda_{\alpha}^{r, \infty}\left(\mathbb{T}^{d}\right)$ for some $0<\alpha<1$ and $1 \leq r \leq \infty$. Then for any $\varepsilon \in(0,1)$, there exists $f_{\varepsilon} \in C^{\infty}\left(\mathbb{T}^{d}\right)$ such that

$$
\left\|f_{\varepsilon}-f\right\|_{r} \leq C \varepsilon^{\alpha}, \quad\left\|\nabla f_{\varepsilon}\right\|_{r} \leq C \varepsilon^{\alpha-1},
$$

where $C>0$ depends on the norm of $f$ in $\Lambda_{\alpha}^{r, \infty}\left(\mathbb{T}^{d}\right)$. Moreover, $\left\|f_{\varepsilon}\right\|_{q} \leq C$ if $\frac{1}{q}>\frac{1}{r}-\frac{\alpha}{d}$, and

$$
\left\|f_{\varepsilon}\right\|_{q} \leq C_{\delta} \varepsilon^{-d\left(\frac{1}{r}-\frac{1}{q}-\frac{\alpha}{d}\right)-\delta},
$$

for any $\delta>0$, if $\frac{1}{q} \leq \frac{1}{r}-\frac{\alpha}{d}$.

Proof. We begin by choosing $g \in C_{0}^{\infty}(B(\mathbf{0}, 1))$ such that $g \geq 0$ and

$$
\int_{\mathbb{R}^{d}} g(\mathbf{x}) d \mathbf{x}=1
$$


For any $\varepsilon \in(0,1)$, let

$$
h_{\varepsilon}(\mathbf{x})=\frac{1}{\varepsilon^{d}} \sum_{\mathbf{n} \in \mathbb{Z}^{d}} g\left(\frac{\mathbf{x}-2 \pi \mathbf{n}}{\varepsilon}\right) .
$$

Clearly $h_{\varepsilon}(\mathbf{x})$ is periodic with respect to the lattice $(2 \pi \mathbb{Z})^{d}$ and $\int_{\mathbb{T}^{d}} h_{\varepsilon}(\mathbf{x}) d \mathbf{x}=1$. Now, given $f \in \Lambda_{\alpha}^{r, \infty}\left(\mathbb{T}^{d}\right)$, we define

$$
f_{\varepsilon}(\mathbf{x})=\int_{\mathbb{T}^{d}} f(\mathbf{y}) h_{\varepsilon}(\mathbf{x}-\mathbf{y}) d \mathbf{y} .
$$

To prove the first inequality in (3.3), we note that

$$
\left|f_{\varepsilon}(\mathbf{x})-f(\mathbf{x})\right| \leq \int_{\mathbb{T}^{d}}|f(\mathbf{x}-\mathbf{y})-f(\mathbf{x})| h_{\varepsilon}(\mathbf{y}) d \mathbf{y} .
$$

By Minkowski's inequality, we have

$$
\begin{aligned}
\left\|f_{\varepsilon}-f\right\|_{r} & \leq \int_{\mathbb{T}^{d}}\|f(\cdot-\mathbf{y})-f(\cdot)\|_{r} h_{\varepsilon}(\mathbf{y}) d \mathbf{y} \\
& \leq C \int_{\mathbb{T}^{d}}\left\{\operatorname{dist}\left(\mathbf{y},(2 \pi \mathbb{Z})^{d}\right)\right\}^{\alpha} h_{\varepsilon}(\mathbf{y}) d \mathbf{y} \\
& \leq \frac{C}{\varepsilon^{d}} \int_{\mathbb{R}^{d}}|\mathbf{y}|^{\alpha} g\left(\frac{\mathbf{y}}{\varepsilon}\right) d \mathbf{y}=C \varepsilon^{\alpha} .
\end{aligned}
$$

To see the second inequality in (3.3), we use

$$
\nabla f_{\varepsilon}(\mathbf{x})=\int_{\mathbb{T}^{d}}(f(\mathbf{x}-\mathbf{y})-f(\mathbf{x})) \nabla h_{\varepsilon}(\mathbf{y}) d \mathbf{y}
$$

The same argument as in (3.8) gives

$$
\left\|\nabla f_{\varepsilon}\right\|_{r} \leq C \int_{\mathbb{T}^{d}}\|f(\cdot-\mathbf{y})-f(\cdot)\|_{r}\left|\nabla h_{\varepsilon}(\mathbf{y})\right| d \mathbf{y} \leq C \varepsilon^{\alpha-1} .
$$

Finally, to estimate $\left\|f_{\varepsilon}\right\|_{q}$, we note that for any $\alpha^{\prime}<\alpha$,

$$
\Lambda_{\alpha}^{r, \infty}\left(\mathbb{T}^{d}\right) \subset \Lambda_{\alpha^{\prime}}^{r, 2}\left(\mathbb{T}^{d}\right) \subset L_{\alpha^{\prime}}^{r}\left(\mathbb{T}^{d}\right) \subset L^{q}\left(\mathbb{T}^{d}\right),
$$

where $\frac{1}{q}=\frac{1}{r}-\frac{\alpha^{\prime}}{d}$ [32]. It then follows from Minkowski's inequality that $\left\|f_{\varepsilon}\right\|_{q} \leq$ $\|f\|_{q} \leq C_{q}$ if $\frac{1}{q}>\frac{1}{r}-\frac{\alpha}{d}$. The remaining case $\frac{1}{q} \leq \frac{1}{r}-\frac{\alpha}{d}$ follows from Young's inequality,

$$
\left\|f_{\varepsilon}\right\|_{q} \leq\|f\|_{p}\left\|h_{\varepsilon}\right\|_{t} \leq C \varepsilon^{-d\left(1-\frac{1}{t}\right)}\|f\|_{p}
$$

where $\frac{1}{q}=\frac{1}{p}+\frac{1}{t}-1$ and $p$ is chosen so that $\frac{1}{p}=\frac{1}{r}-\frac{\alpha}{d}+\frac{\delta}{d}$. This finishes the proof.

Remark 3.11. Letting $0<\alpha<1$, we say $f \in \Lambda_{1+\alpha}^{r, \infty}\left(\mathbb{T}^{d}\right)$ if $f, \nabla f \in L^{r}\left(\mathbb{T}^{d}\right)$ and $\nabla f \in \Lambda_{\alpha}^{r, \infty}\left(\mathbb{T}^{d}\right)$. In this case, if we define $f_{\varepsilon}$ as in (3.6), then $\left\|\nabla f-\nabla f_{\varepsilon}\right\|_{r} \leq C \varepsilon^{\alpha}$ and $\left\|\nabla \nabla f_{\varepsilon}\right\|_{r} \leq C \varepsilon^{\alpha-1}$. Also $\left\|\nabla f_{\varepsilon}\right\|_{q} \leq C$ if $\frac{1}{q}>\frac{1}{r}-\frac{\alpha}{d}$, and

$$
\left\|\nabla f_{\varepsilon}\right\|_{q} \leq C_{\delta} \varepsilon^{-d\left(\frac{1}{r}-\frac{1}{q}-\frac{\alpha}{d}\right)-\delta},
$$

for any $\delta>0$, if $\frac{1}{q} \leq \frac{1}{r}-\frac{\alpha}{d}$. The proof is similar to that of (3.3). Also, note that if $f$ is a real-valued function, then $\inf _{\mathbb{T}^{d}} f \leq \inf _{\mathbb{T}^{d}} f_{\varepsilon}$ and $\sup _{\mathbb{T}^{d}} f_{\varepsilon} \leq \sup _{\mathbb{T}^{d}} f$. These facts will be used in Section 5 . 


\section{The absolute COntinuity of Periodic Dirac operators}

Let $\alpha_{1}, \alpha_{2}, \ldots, \alpha_{d}$ be Hermitian $m \times m$ matrices which satisfy the commutation relations

$$
\alpha_{j} \alpha_{l}+\alpha_{l} \alpha_{j}=2 \delta_{j l} I
$$

where $I=I_{m \times m}$ is the unit matrix. Let $B=\left(b_{j l}\right)_{d \times d}$ be a nonsingular matrix with real constant entries. Consider the Dirac operator

$$
\mathcal{D}(V)=\sum_{j, l=1}^{d} b_{j l} \alpha_{l} D_{j}+V(\mathbf{x}),
$$

acting on $L^{2}\left(\mathbb{R}^{d} ; \mathbb{C}^{m}\right)$, with real $m \times m$ matrix potential $V(\mathbf{x})$. Recall that $D_{j}=$ $-i \partial / \partial x_{j}$. We assume that $V$ is self-adjoint and periodic with respect to some lattice of $\mathbb{R}^{d}$. We also assume that $|V| \in L_{\text {loc }}^{d}\left(\mathbb{R}^{d}\right)$ and $d \geq 3$. By Sobolev imbedding and the Kato-Rellich Theorem, these conditions imply that the Dirac operator $\mathcal{D}(V)$ is self-adjoint on $L^{2}\left(\mathbb{R}^{d} ; \mathbb{C}^{m}\right)$ with domain $H^{1}\left(\mathbb{R}^{d} ; \mathbb{C}^{m}\right)$.

By a change of variables, we may assume that $V$ is periodic with respect to $(2 \pi \mathbb{Z})^{d}$. This is the main reason that we introduce a constant matrix in (4.2). It is well known that using the Floquet-Bloch decomposition, one may reduce the study of the spectrum of a periodic operator on $\mathbb{R}^{d}$ to that of a family of operators on $\mathbb{T}^{d}$. To this end, for $\mathbf{k}=\left(k_{1}, k_{2}, \cdots, k_{d}\right) \in \mathbb{C}^{d}$, we consider the operator

$$
\mathcal{D}(\mathbf{k}, V)=\sum_{j, l=1}^{d} b_{j l} \alpha_{l}\left(D_{j}+k_{j}\right)+V(\mathbf{x})
$$

on $L^{2}\left(\mathbb{T}^{d}, \mathbb{C}^{m}\right)$, with domain $H^{1}\left(\mathbb{T}^{d}, \mathbb{C}^{m}\right)$. Note that $(\mathcal{D}(\mathbf{k}, V))^{*}=\mathcal{D}(\overline{\mathbf{k}}, V)$.

Let $\mathbf{a}, \mathbf{b} \in \mathbb{R}^{d}$ such that $\langle\mathbf{a}, \mathbf{b}\rangle=0$ and (2.6) holds with $A=B B^{T}$. Let $\mathbf{k}=$ $(\delta+i \rho) \mathbf{a}+\mathbf{b}$ where $\delta$ is given by $(2.7), \rho \in \mathbb{R}$ and $|\rho| \geq 2$, as in Section 2 . We denote

$$
\mathcal{D}_{\rho}(V)=\mathcal{D}(\mathbf{k}, V)=\mathcal{D}(((\delta+i \rho) \mathbf{a}+\mathbf{b}), V) .
$$

We will show that under the conditions of Theorem 1.15 , there exists $p \in(1,2]$ such that for any $E \in \mathbb{R}, \mathcal{D}_{\rho}(V)-E I_{m \times m}$ is invertible on $L^{p}\left(\mathbb{T}^{d}, \mathbb{C}^{m}\right)$ if $|\rho|$ is sufficiently large. It follows that the operator $\mathcal{D}_{\rho}(V)$ on $L^{2}\left(\mathbb{T}^{d}, \mathbb{C}^{m}\right)$ has no eigenvalue for $|\rho|$ sufficiently large. By L. Thomas's approach or its variant in [17], this implies that the spectrum of the Dirac operator $\mathcal{D}(V)$ on $L^{2}\left(\mathbb{R}^{d} ; \mathbb{C}^{m}\right)$ is purely absolutely continuous, as in the case of periodic Schrödinger operators.

By replacing $V$ with $V-E I_{m \times m}$, it suffices to establish the invertibility of $\mathcal{D}_{\rho}(V)$. To this end, we follow the previous work in [3] and square the operator.

Proposition 4.5. Assume that $V \in C^{1}\left(\mathbb{T}^{d}\right)$ and $\alpha_{1} V \alpha_{1}=\alpha_{2} V \alpha_{2}=\cdots=\alpha_{d} V \alpha_{d}$. Then

$$
\mathcal{D}(\mathbf{k}, V)\left\{\mathcal{D}(\mathbf{k}, V)-V_{0}\right\}=(\mathbf{D}+\mathbf{k}) B B^{T}(\mathbf{D}+\mathbf{k})^{T}-b_{j l}\left(D_{j} V\right) \alpha_{l}-\left\{V \alpha_{1}\right\}^{2},
$$

where $V_{0}=\left\{\alpha_{1} V \alpha_{1}+V\right\}$.

Proof. First we note that by (4.1),

$\mathcal{D}(\mathbf{k}, V)^{2}=(\mathbf{D}+\mathbf{k}) B B^{T}(\mathbf{D}+\mathbf{k})^{T}+b_{j l}\left(D_{j}+k_{j}\right)\left(V \alpha_{l}+\alpha_{l} V\right)-b_{j l}\left(D_{j} V\right) \alpha_{l}+V^{2}$, 
where the repeated indices are summed from 1 to $d$. Let $V_{0}=\alpha_{1} V \alpha_{1}+V$. Using the condition $\alpha_{1} V \alpha_{1}=\alpha_{2} V \alpha_{2}=\cdots=\alpha_{d} V \alpha_{d}$, it is easy to see that $\alpha_{l} V+V \alpha_{l}=\alpha_{l} V_{0}$. It follows that

$$
\begin{aligned}
\mathcal{D}(\mathbf{k}, V)^{2} & =(\mathbf{D}+\mathbf{k}) B B^{T}(\mathbf{D}+\mathbf{k})^{T}+b_{j l}\left(D_{j}+k_{j}\right) \alpha_{l} V_{0}+V^{2}-b_{j l}\left(D_{j} V\right) \alpha_{l} \\
& =(\mathbf{D}+\mathbf{k}) B B^{T}(\mathbf{D}+\mathbf{k})^{T}+\mathcal{D}(\mathbf{k}, V) V_{0}-b_{j l}\left(D_{j} V\right) \alpha_{l}+V^{2}-V V_{0} \\
& =(\mathbf{D}+\mathbf{k}) B B^{T}(\mathbf{D}+\mathbf{k})^{T}+\mathcal{D}(\mathbf{k}, V) V_{0}-b_{j l}\left(D_{j} V\right) \alpha_{l}-\left(V \alpha_{1}\right)^{2} .
\end{aligned}
$$

From this, formula (4.6) follows easily.

Let $\mathbb{H}_{\rho}=(\mathbf{D}+\mathbf{k}) A(\mathbf{D}+\mathbf{k})^{T}$ where $A=B B^{T}$ and $\mathbf{k}=(\delta+i \rho) \mathbf{a}+\mathbf{b}$. By (4.6), one may write

$$
\begin{aligned}
& \left\{\mathcal{D}_{\rho}(V)\right\}^{-1}= \\
& \left\{b_{j l} \alpha_{l}\left(D_{j}+k_{j}\right)-\alpha_{1} V \alpha_{1}\right\} \mathbb{H}_{\rho}^{-1}\left\{I-b_{j l}\left(D_{j} V\right) \alpha_{l} \mathbb{H}_{\rho}^{-1}-\left\{V \alpha_{1}\right\}^{2} \mathbb{H}_{\rho}^{-1}\right\}^{-1}
\end{aligned}
$$

if the inverses in the right side of (4.7) exist. It follows that if $\left\|b_{j l}\left(D_{j} V\right) \alpha_{l} \mathbb{H}_{\rho}^{-1}\right\|_{p \rightarrow p}$ $+\left\|\left\{V \alpha_{1}\right\}^{2} \mathbb{H}_{\rho}^{-1}\right\|_{p \rightarrow p} \leq 1 / 2$, then

$$
\left\|\mathcal{D}_{\rho}(V)^{-1}\right\|_{p \rightarrow q} \leq C\left\{\left\|(\mathbf{D}+\mathbf{k}) \mathbb{H}_{\rho}^{-1}\right\|_{p \rightarrow q}+\left\|V \alpha_{1} \mathbb{H}_{\rho}^{-1}\right\|_{p \rightarrow q}\right\},
$$

where we used $\|\cdot\|_{p \rightarrow q}$ to denote the operator norm from $L^{p}\left(\mathbb{T}^{d}\right)$ to $L^{q}\left(\mathbb{T}^{d}\right)$. Note that by Theorem 1.11,

$$
\begin{aligned}
\left\|\mathbf{D} \mathbb{H}_{\rho}^{-1}\right\|_{p \rightarrow q} & \leq C|\rho|^{\frac{d-1}{2 r}}(\log |\rho|)^{\frac{d+1}{2 r}}, \\
\left\|\mathbb{H}_{\rho}^{-1}\right\|_{p \rightarrow q} & \leq C|\rho|^{-1+\frac{d-1}{2 r}}(\log |\rho|)^{\frac{d+1}{2 r}},
\end{aligned}
$$

where $\frac{1}{p}+\frac{1}{q}=1$ and $0 \leq \frac{1}{p}-\frac{1}{q}=\frac{1}{r} \leq \frac{1}{d}$. This gives

$$
\begin{aligned}
\left\|b_{j l}\left(D_{j} V\right) \alpha_{l} \mathbb{H}_{\rho}^{-1}\right\|_{p \rightarrow p}+ & \left\|\left(V \alpha_{1}\right)^{2} \mathbb{H}_{\rho}^{-1}\right\|_{p \rightarrow p} \\
& \leq C|\rho|^{-1+\frac{d-1}{2 r}}(\log |\rho|)^{\frac{d+1}{2 r}}\left\{\|\mathbf{D} V\|_{r}+\|V\|_{2 r}^{2}\right\} .
\end{aligned}
$$

In view of (4.8) and (4.9), we also obtain

$$
\left\|\mathcal{D}_{\rho}(V)^{-1}\right\|_{p \rightarrow q} \leq C\left\{|\rho|+\|V\|_{\infty}\right\}|\rho|^{-1+\frac{d-1}{2 r}}(\log |\rho|)^{\frac{d+1}{2 r}}
$$

if the right side of (4.10) is less than $1 / 2$. Theorem 1.15 will follows from estimates (4.10) and (4.11) by an approximation argument.

Proof of Theorem 1.15. Suppose $V \in \Lambda_{\alpha}^{r, \infty}\left(\mathbb{T}^{d}\right)$ for some $r \geq d$ and $1>\alpha>\frac{d-1}{2 r}$. Let $\frac{1}{p}+\frac{1}{q}=1$ and $\frac{1}{p}-\frac{1}{q}=\frac{1}{r}$. We will show that if $|\rho|$ is sufficiently large, $\mathcal{D}_{\rho}(V)$ is invertible on $L^{p}\left(\mathbb{T}^{d} ; \mathbb{C}^{m}\right)$. To do this, we first use Proposition 3.2 to obtain

$$
\begin{aligned}
\left\|V_{\varepsilon}-V\right\|_{r} & \leq C \varepsilon^{\alpha}, \\
\left\|\nabla V_{\varepsilon}\right\|_{r} & \leq C \varepsilon^{\alpha-1},
\end{aligned}
$$

where $V_{\varepsilon}$ is an approximation of $V$ constructed in Proposition 3.2, and the constants in (4.12) depend on $V$. We will also need $\left\|V_{\varepsilon}\right\|_{\infty} \leq C \varepsilon^{-1}$ and $\left\|V_{\varepsilon}\right\|_{2 r}^{2} \leq C \varepsilon^{\alpha-1}$. This follows from estimate (3.4) as well as the assumptions $r \geq d \geq 3$ and $\alpha>\frac{d-1}{2 r}$. 
Next let $\varepsilon=\rho^{-1}$. Since $V_{\varepsilon} \in C^{\infty}\left(\mathbb{T}^{d}\right)$ and $\alpha_{1} V_{\varepsilon} \alpha_{1}=\cdots=\alpha_{d} V_{\varepsilon} \alpha_{d}$, we may apply estimates (4.10) and (4.11) to $V_{\varepsilon}$. This gives

$$
\begin{aligned}
\| b_{j l}\left(D_{j} V_{\varepsilon}\right) & \alpha_{l} \mathbb{H}_{\rho}^{-1}\left\|_{p \rightarrow p}+\right\|\left(V_{\varepsilon} \alpha_{1}\right)^{2} \mathbb{H}_{\rho}^{-1} \|_{p \rightarrow p} \\
& \leq C|\rho|^{-1+\frac{d-1}{2 r}}(\log |\rho|)^{\frac{d+1}{2 r}} \cdot \varepsilon^{\alpha-1} \\
& \leq C(\log |\rho|)^{\frac{d+1}{2 r}}|\rho|^{\frac{d-1}{2 r}-\alpha} \rightarrow 0
\end{aligned}
$$

as $|\rho| \rightarrow \infty$, since $\alpha>\frac{d-1}{2 r}$. By (4.11), this implies that if $|\rho|$ is sufficiently large,

$$
\left\|\mathcal{D}_{\rho}\left(V_{\varepsilon}\right)^{-1}\right\|_{p \rightarrow q} \leq C|\rho|^{\frac{d-1}{2 r}}(\log |\rho|)^{\frac{d+1}{2 r}} .
$$

Finally note that $\mathcal{D}_{\rho}(V)=\mathcal{D}_{\rho}\left(V_{\varepsilon}\right)+\left(V-V_{\varepsilon}\right)$. Using (4.13) and (4.12), we have

$$
\begin{aligned}
\left\|\left(V-V_{\varepsilon}\right) \mathcal{D}_{\rho}\left(V_{\varepsilon}\right)^{-1}\right\|_{p \rightarrow p} & \leq\left\|V-V_{\varepsilon}\right\|_{r}\left\|\mathcal{D}_{\rho}\left(V_{\varepsilon}\right)\right\|_{p \rightarrow q} \\
& \leq C \varepsilon^{\alpha}|\rho|^{\frac{d-1}{2 r}}(\log |\rho|)^{\frac{d+1}{2 r}} \\
& =C|\rho|^{\frac{d-1}{2 r}-\alpha}(\log |\rho|)^{\frac{d+1}{2 r}} \rightarrow 0
\end{aligned}
$$

as $|\rho| \rightarrow \infty$. It follows that if $|\rho|$ is sufficiently large,

$$
\mathcal{D}_{\rho}(V)^{-1}=\mathcal{D}_{\rho}\left(V_{\varepsilon}\right)^{-1}\left\{I+\left(V-V_{\varepsilon}\right) \mathcal{D}_{\rho}\left(V_{\varepsilon}\right)^{-1}\right\}^{-1} .
$$

This shows that $\mathcal{D}_{\rho}(V)$ is invertible on $L^{p}\left(\mathbb{T}^{d} ; \mathbb{C}^{m}\right)$ for sufficiently large $|\rho|$. The proof is finished.

\section{The ABSOlute CONTINUITy OF A SECOND ORDER Elliptic OPERATOR}

Let $\omega(\mathbf{x})$ be a periodic real valued function on $\mathbb{R}^{d}$. Suppose that $\omega \in L^{\infty}\left(\mathbb{R}^{d}\right)$ and $\omega(\mathbf{x}) \geq c_{0}$ for some $c_{0}>0$. Consider the self-adjoint second order elliptic operator

$$
\mathcal{L}_{\omega}=\sum_{j, l} D_{j}\left(\omega(\mathbf{x}) a_{j l} D_{l}\right)
$$

on $L^{2}\left(\mathbb{R}^{d}\right), d \geq 3$, where $A=\left(a_{j l}\right)_{d \times d}$ is a symmetric, positive definite matrix with real constant entries.

By a change of variables, we may assume that $\omega$ is periodic with respect to the standard lattice $(2 \pi \mathbb{Z})^{d}$. We will show in this section that if $\omega \in \Lambda_{1+\alpha}^{r, \infty}\left(\mathbb{T}^{d}\right)$ for some $r \geq d$ and $\alpha>\frac{d-1}{2 r}$, then the spectrum of $\mathcal{L}_{\omega}$ is absolutely continuous. To this end, let $\mathbf{k}=(\delta+i \rho) \mathbf{a}+\mathbf{b} \in \mathbb{C}^{d}$ where $\mathbf{a}, \mathbf{b} \in \mathbb{R}^{d}, \delta \in \mathbb{R}$ are fixed as in Section 2 and $\rho \geq 2$ is a parameter. We consider a family of operators

$$
\mathcal{L}_{\omega}(\mathbf{k})=\sum_{j, l}\left(D_{j}+k_{j}\right)\left(\omega(\mathbf{x}) a_{j l}\left(D_{l}+k_{l}\right)\right)
$$

on $L^{2}\left(\mathbb{T}^{d}\right)$. By L. Thomas's approach, it suffices to show that the family of operators $\left\{\mathcal{L}_{\omega}(\mathbf{k}): \rho \geq 2\right\}$ has no common eigenvalues.

Proof of Theorem 1.22. Suppose that $\left\{\mathcal{L}_{\omega}(\mathbf{k}): \rho \geq 2\right\}$ has a common eigenvalue $E$. That is, for each $\rho \geq 2$, there exists $\psi_{\rho} \in \operatorname{Domain}\left(\mathcal{L}_{w}(\mathbf{k})\right) \subset H^{1}\left(\mathbb{T}^{d}\right)$ such that

$$
\left\|\psi_{\rho}\right\|_{2}=1 \quad \text { and } \quad \mathcal{L}_{\omega}(\mathbf{k}) \psi_{\rho}=E \psi_{\rho} .
$$

Let

$$
\varphi_{\rho}=\widetilde{\omega}^{1 / 2} \psi_{\rho},
$$


where $\widetilde{\omega} \geq c_{0}$ is a real valued positive function in $C^{\infty}\left(\mathbb{T}^{d}\right)$ to be determined. Then

$$
\begin{aligned}
(\mathbf{D}+\mathbf{k}) A(\mathbf{D}+\mathbf{k})^{T} \varphi_{\rho} & =\sum_{j, l} a_{j l}\left\{\frac{D_{j} \widetilde{\omega}}{\widetilde{\omega}}-\frac{D_{j} \omega}{\omega}\right\}\left(D_{l}+k_{l}\right) \varphi_{\rho}+\frac{E}{\omega} \varphi_{\rho} \\
& +\left(\frac{1}{2 \widetilde{\omega}} \sum_{j, l} a_{j l} D_{j} D_{l} \widetilde{\omega}+\frac{1}{2 \omega \widetilde{\omega}} \sum_{j, l} a_{j l}\left(D_{j} \omega\right)\left(D_{l} \widetilde{\omega}\right)\right) \varphi_{\rho} \\
& -\left(\frac{3}{4 \widetilde{\omega}^{2}} \sum_{j, l} a_{j l}\left(D_{j} \widetilde{\omega}\right)\left(D_{l} \widetilde{\omega}\right)\right) \varphi_{\rho} .
\end{aligned}
$$

It follows that for any $p>1$, we have

$$
\begin{aligned}
\left\|(\mathbf{D}+\mathbf{k}) A(\mathbf{D}+\mathbf{k})^{T} \varphi_{\rho}\right\|_{p} \leq & C\left\|\sum_{j, l}\left|\frac{D_{j} \widetilde{\omega}}{\widetilde{\omega}}-\frac{D_{j} \omega}{\omega}\right|\left(D_{l}+k_{l}\right) \varphi_{\rho}\right\|_{p}+C\left\|\varphi_{\rho}\right\|_{p} \\
& +C\left\{\left\||\nabla \nabla \widetilde{\omega}| \varphi_{\rho}\right\|_{p}+\left\||\nabla \omega||\nabla \widetilde{\omega}| \varphi_{\rho}\right\|_{p}\right\} \\
& +C\left\||\nabla \widetilde{\omega}|^{2} \varphi_{\rho}\right\|_{p} .
\end{aligned}
$$

Suppose $\omega \in \Lambda_{1+\alpha}^{r, \infty}\left(\mathbb{T}^{d}\right)$ for some $r \geq d$ and $1>\alpha>\frac{d-1}{2 r}$. Choose $(p, q)$ so that $\frac{1}{p}+\frac{1}{q}=1$ and $\frac{1}{p}-\frac{1}{q}=\frac{1}{r}$, i.e., $p=\frac{2 r}{r+1}$ and $q=\frac{2 r}{r-1}$. Let $\mathbb{H}_{\rho}=(\mathbf{D}+\mathbf{k}) A(\mathbf{D}+\mathbf{k})^{T}$. Recall that by Theorem 1.11,

$$
\begin{aligned}
\|\varphi\|_{q} & \leq C|\rho|^{-1+\frac{d-1}{2 r}}(\log |\rho|)^{\frac{d+1}{2 r}}\left\|\mathbb{H}_{\rho} \varphi\right\|_{p}, \\
\|(\mathbf{D}+\mathbf{k}) \varphi\|_{q} & \leq C|\rho|^{\frac{d-1}{2 r}}(\log |\rho|)^{\frac{d+1}{2 r}}\left\|\mathbb{H}_{\rho} \varphi\right\|_{p} .
\end{aligned}
$$

Let $\varepsilon=|\rho|^{-1}$ and choose $\widetilde{\omega}=\omega_{\varepsilon} \in C^{\infty}\left(\mathbb{T}^{d}\right)$ where $\omega_{\varepsilon}$ is an approximation of $\omega$ constructed in Proposition 3.2. By Remark 3.11, we have $\widetilde{\omega} \geq c_{0}$ and

$$
\|\nabla \widetilde{\omega}-\nabla \omega\|_{r} \leq C \varepsilon^{\alpha}, \quad\|\nabla \nabla \widetilde{\omega}\|_{r} \leq C \varepsilon^{\alpha-1} .
$$

Moreover, $\|\nabla \widetilde{\omega}\|_{t} \leq C$ if $\frac{1}{t}>\frac{1}{r}-\frac{\alpha}{d}$, and

$$
\|\nabla \widetilde{\omega}\|_{t} \leq C_{\delta} \varepsilon^{-d\left(\frac{1}{r}-\frac{1}{t}-\frac{\alpha}{d}\right)-\delta} \quad \text { for any } \delta>0
$$

if $\frac{1}{t} \leq \frac{1}{r}-\frac{\alpha}{d}$. These estimates, together with (5.7), will be used to control the right side of (5.6).

To do this, we first note that

$$
\left|\frac{\nabla \widetilde{\omega}}{\widetilde{\omega}}-\frac{\nabla \omega}{\omega}\right| \leq C\{|\nabla \widetilde{\omega}-\nabla \omega|+|\nabla \widetilde{\omega}||\omega-\widetilde{\omega}|\},
$$

and $\||\nabla \widetilde{\omega}||\omega-\widetilde{\omega}|\|_{r} \leq\|\nabla \widetilde{\omega}\|_{r_{1}}\|\omega-\widetilde{\omega}\|_{r_{2}}$, where $\frac{1}{r}=\frac{1}{r_{1}}+\frac{1}{r_{2}}$ and $r<r_{1}, r_{2}<\infty$. By Sobolev's inequality and the observation that $\int_{\mathbb{T}^{d}}(\omega-\widetilde{\omega}) d \mathbf{x}=0$, for any $r<r_{2}<\infty$,

$$
\|\omega-\widetilde{\omega}\|_{r_{2}} \leq C\|\nabla \omega-\nabla \widetilde{\omega}\|_{d} \leq C\|\nabla \omega-\nabla \widetilde{\omega}\|_{r} \leq C \varepsilon^{\alpha} .
$$

We then choose $r_{1}>r$ so close to $r$ that $\|\nabla \widetilde{\omega}\|_{r_{1}} \leq C$. We obtain

$$
\begin{aligned}
\left\|\frac{\nabla \widetilde{\omega}}{\widetilde{\omega}}-\frac{\nabla \omega}{\omega}\right\|_{r} & \leq C\left\{\|\nabla \omega-\nabla \widetilde{\omega}\|_{r}+\|\mid \nabla \widetilde{\omega}\| \omega-\widetilde{\omega} \|_{r}\right\} \\
& \leq C \varepsilon^{\alpha} .
\end{aligned}
$$


It follows that

$$
\begin{aligned}
\left\|\left\{\frac{D_{j} \widetilde{\omega}}{\widetilde{\omega}}-\frac{D_{j} \omega}{\omega}\right\}\left(D_{l}+k_{l}\right) \varphi_{\rho}\right\|_{p} & \leq\left\|\frac{D_{j} \widetilde{\omega}}{\widetilde{\omega}}-\frac{D_{j} \omega}{\omega}\right\|_{r}\left\|\left(D_{l}+k_{l}\right) \varphi_{\rho}\right\|_{q} \\
& \leq C \varepsilon^{\alpha}|\rho|^{\frac{d-1}{2 r}}(\log |\rho|)^{\frac{d+1}{2 r}}\left\|\mathbb{H}_{\rho} \varphi_{\rho}\right\|_{p} \\
& \leq C \rho^{\frac{d-1}{2 r}-\alpha}(\log |\rho|)^{\frac{d+1}{2 r}}\left\|\mathbb{H}_{\rho} \varphi_{\rho}\right\|_{p} .
\end{aligned}
$$

Next we wish to show that

$$
\|\nabla \nabla \widetilde{\omega}\|_{r}+\||\nabla \omega||\nabla \widetilde{\omega}|\|_{r}+\left\||\nabla \widetilde{\omega}|^{2}\right\|_{r} \leq C \varepsilon^{\alpha-1} .
$$

This, together with (5.6), (5.12) and Hölder's inequality, implies that

$$
\left\|\mathbb{H}_{\rho} \varphi_{\rho}\right\|_{p} \leq C|\rho|^{\frac{d-1}{2 r}-\alpha}(\log |\rho|)^{\frac{d+1}{2 r}}\left\|\mathbb{H}_{\rho} \varphi_{\rho}\right\|_{p}
$$

for any $\rho \geq 2$. Since $\alpha>\frac{d-1}{2 r}$, estimate (5.14) gives us a contradiction, provided we can show that $\mathbb{H}_{\rho} \varphi_{\rho} \in L^{p}\left(\mathbb{T}^{d}\right)$. But this is not hard to do. Indeed, since $\varphi_{\rho} \in$ $H^{1}\left(\mathbb{T}^{d}\right)$ and $\widetilde{\omega} \in C^{\infty}\left(\mathbb{T}^{d}\right)$, in view of (5.6), it suffices to prove that $|\nabla \omega|\left|\nabla \varphi_{\rho}\right| \in$ $L^{p}\left(\mathbb{T}^{d}\right)$. Furthermore, by Hölder's inequality, we only need to show that $\nabla \omega \in$ $L^{t}\left(\mathbb{T}^{d}\right)$, where $\frac{1}{t}=\frac{1}{p}-\frac{1}{2}=\frac{1}{2 r}$. To this end, we use the Sobolev imbedding (3.10). It shows that $\nabla \omega \in L^{s}\left(\mathbb{T}^{d}\right)$ for any $s$ such that $\frac{1}{s}>\frac{1}{r}-\frac{\alpha}{d}$. Using $\alpha>\frac{d-1}{2 r}$, one may verify that $\frac{1}{2 r}>\frac{1}{r}-\frac{\alpha}{d}$.

It remains to prove estimate (5.13). To do this, we note that the estimate $\|\nabla \nabla \widetilde{\omega}\|_{r} \leq C \varepsilon^{\alpha-1}$ is already in (5.8). Also, if $\frac{1}{r}=\frac{1}{t_{1}}+\frac{1}{t_{2}}$

$$
\begin{aligned}
\||\nabla \omega||\nabla \widetilde{\omega}|\|_{r}+\left\||\nabla \widetilde{\omega}|^{2}\right\|_{r} & \leq\|\nabla \omega\|_{t_{1}}\|\nabla \widetilde{\omega}\|_{t_{2}}+\|\nabla \widetilde{\omega}\|_{t_{1}}\|\nabla \widetilde{\omega}\|_{t_{2}} \\
& \leq 2\|\nabla \omega\|_{t_{1}}\|\nabla \widetilde{\omega}\|_{t_{2}} .
\end{aligned}
$$

We may assume $\frac{1}{r}-\frac{\alpha}{d}>0$ (for otherwise, we would have $\|\nabla \omega\|_{t}+\|\nabla \omega\|_{t} \leq C_{t}$ for any $t<\infty$, and the desired estimate would follow easily). Choose $t_{1}>r$ so that $\frac{1}{t_{1}}>\frac{1}{r}-\frac{\alpha}{d}$. If we also have $\frac{1}{t_{2}}>\frac{1}{r}-\frac{\alpha}{d}$, then the left side of (5.15) is bounded by a constant independent of $\varepsilon$.

Finally, suppose $\frac{1}{t_{2}} \leq \frac{1}{r}-\frac{\alpha}{d}$. Then the right side of (5.15) is bounded by

$$
C\|\nabla \widetilde{\omega}\|_{t_{2}} \leq C \varepsilon^{-d\left(\frac{1}{r}-\frac{1}{t_{2}}-\frac{\alpha}{d}\right)-\delta}=C \varepsilon^{-d\left(\frac{1}{t_{1}}-\frac{\alpha}{d}\right)-\delta} .
$$

We need to find $t_{1}>r$ and $\delta>0$ so that $\frac{1}{t_{1}}>\frac{1}{r}-\frac{\alpha}{d}$ and $d\left(\frac{1}{t_{1}}-\frac{\alpha}{d}\right)+\delta \leq 1-\alpha$. This is clearly possible since we assume that $r \geq d$. The proof is now complete.

\section{REFERENCES}

[1] M.Sh. Birman and T.A. Suslina, Two-dimensional periodic magnetic Hamiltonian is absolutely continuous, Algebra i Analiz 9 (1) (1997), 32-48; English transl. in St. Petersburg Math. J. 9 (1) (1998), 21-32. MR1458417 (98g:47038)

[2] M.Sh. Birman and T.A. Suslina, Absolute continuity of the two-dimensional periodic magnetic Hamiltonian with discontinuous vector-valued potential, Algebra i Analiz 10 (4), 1-36; English transl. in St. Petersburg Math. J. 10 (4) (1999), 579-601. MR1654063 (99k:81060)

[3] M.Sh. Birman and T.A. Suslina, The periodic Dirac operator is absolutely continuous, Integral Equations Operator Theory 34 (4) (1999), 377-395. MR1702229 (2000h:47068)

[4] M.Sh. Birman and T.A. Suslina, A periodic magnetic Hamiltonian with a variable metric: The problem of absolute continuity, Algebra i Analiz 11 (2) (1999), 1-40; English transl. in. St. Petersburg Math. J. 11 (2) (2000), 203-232. MR1702587 (2000i:35026)

[5] M.Sh. Birman, T.A. Suslina, and R.G. Shterenberg, Absolute continuity of the twodimensional Schrödinger operator with potential supported on a periodic system of curves, Algebra i Analiz 12 (6) (2000), 140-177; English transl. in. St. Petersburg Math. J. 12 (6) (2001), 983-1012. MR1816514 (2002k:35227) 
[6] L.I. Danilov, On the spectrum of the Dirac operator with periodic potential in $\mathbb{R}^{n}$, Teoret. Mat. Fiz. 85 (1) (1990), 41-53; English transl. in Theoret. Math. Phys. 85 (1) (1991), 10391048. MR1083951 (92a:35119)

[7] L.I. Danilov, Resolvent estimates and the spectrum of the Dirac operator with a periodic potential, Teoret. Mat. Fiz 103 (1) (1995), 3-22; English transl. in. Theoret. Math. Phys. 103 (1) (1995), 349-365. MR1470934 (98f:35112)

[8] L.I. Danilov, On the spectrum of the periodic Dirac operator, Teoret. Mat. Fiz. 124 (1) (2000), 3-17; English transl. in. Theoret. Math. Phys. 124 (1) (2000), 859-871. MR1821309 (2002b:81028)

[9] L.I. Danilov, Absolute continuity of the spectrum of a periodic Dirac operator, Differ. Uravn. 36 (2) (2000), 233-240; English transl. in. Differ. Equ. 36 (2) (2000), 262-271. MR1773794 (2001f:47082)

[10] L.I. Danilov, On the spectrum of the two-dimentional periodic Schrödinger operator, Teoret. Mat. Fiz. 134 (2003), 447-459; English transl. in Theoret. Math. Phys. 134 (2003), 392-403. MR2001818 (2004j:35210)

[11] L.I. Danilov, On the absolute continuity of the spectrum of a periodic Schrödinger operator, Mat. Zametki 73 (2003), no. 1, 49-62; English transl. in Math. Notes 73 (1) (2003), 46-57. MR1993539 (2004f:35130)

[12] L.I. Danilov, On absence of eigenvalues in the spectrum of generalized two-dimensional periodic Dirac operators, Algebra i Analiz 17 (3) (2005), 47-80. MR2167843 (2006m:35261)

[13] L. Friedlander, On the spectrum of a class of second order periodic elliptic differential operators, Commun. Math. Phys. 229 (2002), 49-55. MR1917673 (2003k:35179)

[14] L. Grafakos, Classical and Modern Fourier Analysis, Pearson Education, Inc., 2004.

[15] C.E. Kenig, Restriction theorems, Carleman estimates, uniform Sobolev inequalities and unique continuation, Lecture Notes in Math. 1384 (1989), 69-90. MR1013816 (90m:35016)

[16] C.E. Kenig, A. Ruiz, and C.D. Sogge, Uniform Sobolev inequalities and unique continuation for second order constant coefficient differential operators, Duke Math. J. 55 (2) (1987), 329-347. MR894584 (88d:35037)

[17] P. Kuchment, Floquet Theory for Partial Differential Equations, Birkhäuser Verlag, 1993. MR1232660 (94h:35002)

[18] P. Kuchment and S. Levendorskii, On the structure of spectra of periodic elliptic operators, Trans. Amer. Math. Soc. 354 (2) (2002), 537-569. MR1862558 (2002g:35163)

[19] I.S. Lapin, Absolute continuity of the spectra of two-dimensional periodic magnetic Schrödinger operator and Dirac operator with potentials in the Zygmund class, Function theory and phase transitions. J. Math. Sci. (New York) 106 (3) (2001), 2952-2974. MR1906028 (2003h:35182)

[20] A. Morame, Absence of singular spectrum for a perturbation of a two-dimensional LaplaceBeltrami operator with periodic electro-magnetic potential, J. Phys. A: Math. Gen. 31 (1998), 7593-7601. MR1652918 (99i:81039)

[21] M. Reed and B. Simon, Methods of Modern Mathematical Physics,, vol. IV, Academic Press, 1978. MR0493422 (58:12430a)

[22] Z. Shen, On absolute continuity of the periodic Schrödinger operators, Internat. Math. Res. Notices 2001 (1) (2001), 1-31. MR1809495 (2002a:47078)

[23] Z. Shen, Absolute continuity of generalized periodic Schrödinger operators, Contemp. Math. 277 (2001), 113-126. MR1840430 (2002j:35078)

[24] Z. Shen, Absolute continuity of periodic Schrödinger operators with potentials in the Kato class, Illinois J. Math. 45 (3) (2001), 873-893. MR1879241 (2002m:35036)

[25] Z. Shen, The periodic Schrödinger operator with potentials in the Morrey class, J. Funct. Anal. 193 (2002), 314-345. MR1929505 (2003k:47071)

[26] R. G. Shterenberg, Absolute continuity of a two-dimensional magnetic periodic Schrödinger operator with electric potential of measure derivative type, Zap. Nauchn. Sem. S.-Peterburg. Otdel. Mat. Inst. Steklov. (POMI) 271 (2000), Kraev. Zadachi Mat. Fiz. i Smezh. Vopr. Teor. Funkts. 31, 276-312, 318; English transl. in J. Math. Sci. (N.Y.) 115 (6) (2003), 2862-2882. MR1810620 (2002m:35171)

[27] R. G. Shterenberg, Absolute continuity of the spectrum of the two-dimensional magnetic periodic Schrödinger operator with positive electric potential., Proceedings of the St. Petersburg Mathematical Society IX (2003), 191-221. MR2018378 (2005d:35185) 
[28] R. G. Shterenberg, Absolute continuity of the spectrum of the two-dimensional periodic Schrödinger operator with strongly subordinate magnetic potential (in Russian), Zap. Nauchn. Sem. S.-Peterburg. Otdel. Mat. Inst. Steklov. (POMI) 303 (2003). MR2018378 (2005d:35185)

[29] R.G. Shterenberg and T.A. Suslina, Absolute continuity of the spectrum for the Schrödinger operator with the potential concentrated on a periodic system of hypersurfaces, St. Petersburg Math. J. 13 (2002), 859-891. MR1882869 (2002m:35172)

[30] A.V. Sobolev, Absolute continuity of the periodic magnetic Schrödinger operator, Invent. Math. 137 (1) (1999), 85-112. MR1703339 (2000g:35028)

[31] C.D. Sogge, Concerning the $L^{p}$ norm of spectral clusters of second order elliptic operators on compact manifolds, J. Funct. Anal. 77 (1) (1988), 123-138. MR930395 (89d:35131)

[32] E.M. Stein, Singular Integrals and Differentiability Properties of Functions, Princeton Univ. Press, 1970. MR0290095 (44:7280)

[33] E.M. Stein and G. Weiss, Introduction to Fourier Analysis on Euclidean Spaces, Princeton Univ. Press, 1971. MR0304972 (46:4102)

[34] T. Suslina, Absolute continuity of the spectrum of periodic operators of mathematical physics, Journées Equations aux Dérivées Partielles (2000), 1-13. MR1775694 (2001f:35295)

[35] L.E. Thomas, Time dependent approach to scattering from impurities in a crystal, Comm. Math. Phys. 33 (1973), 335-343. MR0334766 (48:13084)

[36] M. Tikhomirov and N. Filonov, Absolute continuity of an "even" periodic Schrödinger operator with non-smooth coefficients, Algebra i Analiz 16 (3) (2004), 201-210; English transl. in St. Petersburg Math. J. 16 (3) (2005), 583-589. MR2083570 (2005f:35056)

[37] T. Wolff, A property of measures in $\mathbb{R}^{n}$ and an application to unique continuation, Geom. Funct. Anal. 2 (1992), 225-284. MR1159832 (93c:35015)

Department of Mathematics, University of Kentucky, Lexington, Kentucky 40506

E-mail address: shenz@ms.uky.edu

Department of Mathematics, Lanzhou University, Lanzhou, Gansu, 730000, People's RePUBlic OF CHINA

E-mail address: zhaoph@lzu.edu.cn 